\title{
Convex relaxations in the optimal control of electrified vehicles
}

\author{
Nikolce Murgovski, Lars Johannesson, Xiaosong Hu, Bo Egardt, Jonas Sjöberg
}

\begin{abstract}
When controlling the energy flow among multiple power sources in electrified powertrains, the typically nonconvex set of the original formulation is relaxed to a convex super-set, and the problem is then approached by the means of convex optimization. In this paper we show that when using the backward simulation approach, where vehicle velocity is equal to the reference velocity, the global optimum of the original nonconvex problem can be obtained by solving a relaxed convex problem. When vehicle velocity is kept as a state in the problem, in the so called forward simulation approach, we provide a condition for which, when satisfied, the solution of the relaxed problem will provide the solution of the non-relaxed problem.
\end{abstract}

\section{INTRODUCTION}

The usage of convex optimization in vehicular technology is being spread to several applications. In [1], [2] convex optimization is used for minimum time optimal control, while partial convexification for predictive cruise control of conventional vehicles has been employed by [3]. In the context of hybrid electric vehicles (HEV) [4], convex optimization is being used for performance assessment [5], [6], [7], optimal energy management [8], [9], [10], [11], and powertrain sizing [12], [13], [14], [15], [16], [17], [18], [19]. Main motivations for the penetration of convex optimization are the short computational time, the certificate for optimality and the availability of publicly accessible solvers, such as ECOS [20], SeDuMi [21] and SDPT3 [22].

Many of these problems, however, are not convex in their original settings. The typical convexification step is to perform relaxations, such that the non-convex set is replaced by a convex superset. The goal is to obtain the solution of the original non-convex problem by efficiently solving a relaxed convex problem.

A typical example of convex relaxations, in the scope of HEVs, is letting the relaxed problem throw away energy. When using a backward simulation approach, where vehicle speed is assumed equal to the reference, it is logically argued that the solution of the non-convex problem can be obtained by solving the relaxed problem, since the algorithm will make sure that energy is not wasted at the optimum [12], [13]. A more rigorous proof that the relaxations are tight at the optimum has been provided by [16].

Complementing the work of [16], this paper provides a more detailed proof that the global optimum of the nonconvex backward simulation problem can be obtained by

This work was supported in part by the Swedish Energy Agency.

The authors are with the Department of Signals and Systems, Chalmers University of Technology, Gothenburg, Sweden. L. Johannesson is also with Viktoria Swedish ICT, Gothenburg, Sweden. nikolce.murgovski@chalmers.se, larsjo@chalmers.se, xiaosong@chalmers.se, bo.egardt@chalmers.se, jonas.sjoberg@chalmers.se solving a relaxed convex problem. We show that a special case exists where wasting energy is unavoidable and the relaxation may not be tight, although the global optimum can still be obtained by solving the relaxed problem.

In addition, this paper investigates the forward simulation approach, where vehicle speed is kept as an optimization variable in the problem. We show that for the forward simulation approach, relaxations should be considered with care, since it is not always possible to retrieve the solution of the non-relaxed problem by solving a relaxed convex problem. However, we provide a condition, which if satisfied, will guarantee that the global optimum of the non-relaxed problem can be obtained from the solution of the relaxed problem.

\section{Vehicle MODELING AND OPTIMIZATION OBJECTIVE}

We consider an optimal vehicle controller that manages energy flow between powertrain components, such that the amount of fuel needed to drive a certain road segment is minimized. The driving segment is here referred to as driving cycle, described by a desired reference velocity $v_{r}$ and a road gradient $\alpha$.

Maintaining the desired velocity requires a certain longitudinal force that overcomes dissipative forces due to aerodynamic drag and rolling resistance

$$
F_{\mathrm{Vd}}(\cdot)=\frac{\rho_{a} c_{d} A_{f}}{2} v^{2}+m g c_{r} \cos \alpha
$$

where $\rho_{a}$ is air density, $c_{d}$ is drag coefficient, $A_{f}$ is vehicle's frontal area, $m$ is vehicle's mass, $v$ is longitudinal vehicle velocity, $c_{r}$ is rolling resistance coefficient and $g$ is gravity. The longitudinal power

$$
P_{\mathrm{V}}(\cdot)=\left(m \frac{d v}{d t}+m g \sin \alpha+F_{\mathrm{Vd}}(\cdot)\right) v
$$

could be provided in several ways, depending on the powertrain configuration. For this study we have chosen a hybrid electric vehicle in a series powertrain configuration [4], depicted in Fig. 1. It is straight forward, however, to extend the forthcoming strategies to other types of electrified vehicles, and even conventional vehicles.

The series HEV powertrain provides the longitudinal power by an electric machine (EM), mechanically connected to the wheels without a transmission. Denoting the mechanical EM power by $P_{\mathrm{M}}$, the following relation holds

$$
P_{\mathrm{M}}=P_{\mathrm{V}}(\cdot)+P_{\text {brk }}
$$

where $P_{\mathrm{brk}}$ is power applied to the braking pads. Note that, in an electrified vehicle it is preferable to use the EM to brake 


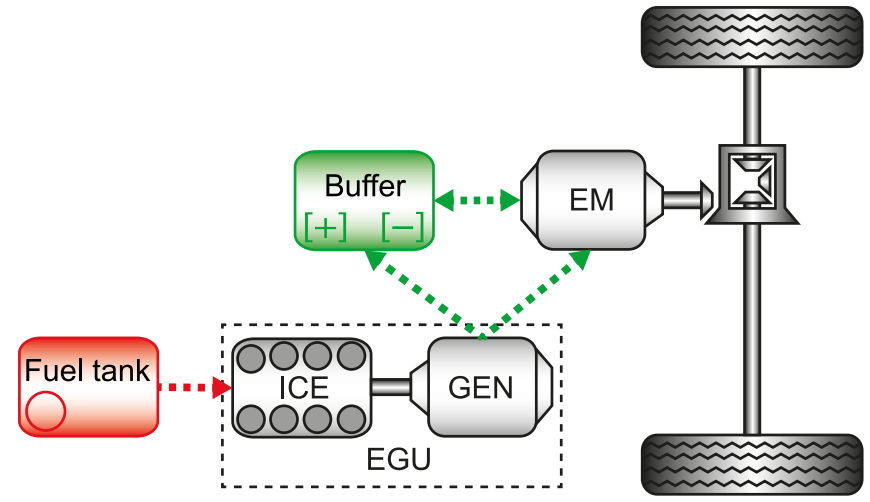

Fig. 1. Series HEV powertrain model. The vehicle is propelled by an electric machine (EM), which obtains electrical energy from an engine generator unit (EGU), or a battery. When EM operates as a generator, braking energy is converted to (and stored as) electrical energy in the battery.

the vehicle by recuperating braking energy, which is stored in the battery for a later use. When operated in motoring mode, the EM obtains electrical energy from an engine generator unit (EGU) and the battery, which can be described by the following equation

$$
P_{\mathrm{G}}+P_{\mathrm{B}}=P_{\mathrm{Bd}}(\cdot)+P_{\mathrm{M}}+P_{\mathrm{Md}}(\cdot)+P_{\mathrm{A}}
$$

where $P_{\mathrm{G}}$ is electrical power generated by the EGU, $P_{\mathrm{B}}$ is internal battery power, $P_{\mathrm{A}} \geq 0$ is power consumed by auxiliary devices, and $P_{\mathrm{Bd}}(\cdot), P_{\mathrm{Md}}(\cdot)$ are dissipative powers of the battery and the EM. The losses of the inverter and the final differential gear are included within $P_{\mathrm{Md}}(\cdot)$.

Taking into account the relations (1)-(4) and the objective of minimizing fuel energy, the optimization problem can be formulated as

$$
\begin{aligned}
& \min J(\cdot)=\int_{0}^{t_{f}}\left(P_{\mathrm{G}}+P_{\mathrm{Gd}}(\cdot)\right) d t \\
& \text { s.t. }\left(\forall t \in\left[0, t_{f}\right]\right) \\
& P_{\mathrm{M}}=P_{\mathrm{V}}(\cdot)+P_{\mathrm{brk}} \\
& P_{\mathrm{G}}+P_{\mathrm{B}}=P_{\mathrm{Bd}}(\cdot)+P_{\mathrm{M}}+P_{\mathrm{Md}}(\cdot)+P_{\mathrm{A}} \\
& \frac{d E_{\mathrm{B}}}{d t}=-P_{\mathrm{B}} \\
& t_{f} \leq t_{\max } \\
& v(0)=v\left(t_{f}\right)=v_{0} \\
& v \in\left[v_{\min }, v_{\max }\right] \\
& E_{\mathrm{B}}(0)=E_{\mathrm{B}}\left(t_{f}\right)=E_{\mathrm{B} 0} \\
& E_{\mathrm{B}} \in\left[E_{\mathrm{Bmin}}, E_{\mathrm{Bmax}}\right] \\
& P_{\mathrm{B}} \in\left[P_{\mathrm{B} \min }(\cdot), P_{\mathrm{Bmax}}(\cdot)\right] \\
& P_{\mathrm{G}} \in\left[0, \sigma_{\mathrm{G}} P_{\mathrm{Gmax}}\right] \\
& P_{\mathrm{M}} \in\left[P_{\mathrm{M} \min }(\cdot), P_{\mathrm{M} \max }(\cdot)\right] \\
& P_{\mathrm{brk}} \geq 0 .
\end{aligned}
$$

where $E_{\mathrm{B}}$ is energy in the battery, $P_{\mathrm{Gd}}(\cdot)$ is EGU dissipative power and $\sigma_{\mathrm{G}}$ is a binary signal that governs the engine on/off state. Initial and final battery energy and vehicle's kinetic energy are conserved by (5f) and (5h), and travel time is restrained below $t_{\max }$. Constraints are imposed on the control and state variables.

The notation $(\cdot)$ indicates a function of decision variables. The formulation (5) considers many decision variables, $E_{\mathrm{B}}$, $P_{\mathrm{B}}, P_{\mathrm{G}}, P_{\mathrm{M}}, P_{\mathrm{brk}}, v, t_{f}$, although alternative formulations exist with fewer variables. An example of such formulation is discussed later, in Section III-A. The terms $P_{\mathrm{A}}, E_{\mathrm{Bmin}}$, $E_{\mathrm{Bmax}}, P_{\mathrm{Gmax}}$ and $t_{\max }$ are known scalar values, while the signals denoting energy, power, force, speed, engine on/off and road gradient are functions of time. Thus, the constraints in (5) are imposed at each time instant along the driving cycle.

The longitudinal vehicle power has been defined in (2), where it is evident that $P_{\mathrm{V}}(\cdot)=P_{\mathrm{V}}(v)$ is nonlinear and non-convex in $v$. A description of the remaining functions in problem (5) will be given later, in Section IV-A. These function, however, are known to have the following properties:

1) $P_{\mathrm{Gd}}(\cdot)=P_{\mathrm{Gd}}\left(P_{\mathrm{G}}, \sigma_{\mathrm{G}}\right)$ is convex and monotonically increasing in $P_{\mathrm{G}}$.

2) $P_{\mathrm{Bd}}(\cdot)=P_{\mathrm{Bd}}\left(P_{\mathrm{B}}, E_{\mathrm{B}}\right)$ is nonlinear and convex in $P_{\mathrm{B}}$ and $E_{\mathrm{B}}$.

3) $P_{\mathrm{Bmin}}(\cdot)=P_{\mathrm{Bmin}}\left(E_{\mathrm{B}}\right)$ is convex in $E_{\mathrm{B}} ; P_{\mathrm{B} \max }(\cdot)=$ $P_{\mathrm{Bmax}}\left(E_{\mathrm{B}}\right)$ is concave in $E_{\mathrm{B}}$.

4) $P_{\mathrm{Md}}(\cdot)=P_{\mathrm{Md}}\left(P_{\mathrm{M}}, v\right)$ is convex in $P_{\mathrm{M}}$ and $v$, and nonlinear, in general.

More details on the convexity of these functions can be found in [12], [23], [24], [4], [13], [15]. A convex and nondecreasing EGU model has been proposed by [12], based on the EGU models of [25]. The convexity of buffer losses and power limits have been established by [23], [16] and hold both for supercapacitors and batteries modeled with linear relationship between open circuit voltage and state of charge. Quadratic EM model, convex in $P_{\mathrm{M}}$, has been widely used in literature; see, e.g., [4] and references therein. The convexity of $P_{\mathrm{Md}}(\cdot)$ with respect to $v$ is here a mild requirement, as for example, an optimization approach is discussed in Section III where convexity in $v$ is not required. Nevertheless, more details on the EM convexity, in both power and speed, can be found in [15]. The engine on/off state $\sigma_{\mathrm{G}}$ is regarded as an external signal, since obtaining its optimal value is not in the context of this study. Interested readers are referred to [26], [27] for optimization of $\sigma_{\mathrm{G}}$ by iteratively solving convex problems.

The optimization problems discussed in the rest of the paper are to be solved by approximating derivatives and integrals by affine relations.

\section{CONVEX RELAXATION FOR BACKWARD SIMULATION}

In order to reduce computational complexity and simultaneously eliminate the non-convex function $P_{\mathrm{V}}(\cdot)$, the problem of optimal energy management of electrified powertrains is typically optimized in the so called backward simulation approach [28]. The approach assumes that the vehicle follows exactly the reference velocity, i.e. $v=v_{r}$, which reduces the computational complexity in optimization by removing the state variable $v$ from the problem. However, it requires an 
additional preprocessing step that ensures that the problem is feasible, which can be achieved by, e.g., pre-filtering the reference velocity [29], [7].

The resulting optimization problem is similar to (5), with the difference that $t_{f}$ is fixed and $v, P_{\mathrm{V}}, P_{\mathrm{Mmin}}$ become known signals of time. Thus, the constraints $(5 \mathrm{e}),(5 \mathrm{~g})$ are removed from the problem, while convexity of $P_{\mathrm{Md}}(\cdot)$ with respect to $v$ is not required. Similarly, the upper bound $P_{\text {Mmax }}$ can be removed from the problem, since the pre-filtering step ensures that this constraint is not violated. However, the feasible set of the resulting optimization problem is not convex. It is well known that an equality constraint between two functions defines a convex set only if the functions are affine [30], which is not the case with (5c) where the righthand side is nonlinear. In fact, two nonlinear functions, one convex and the other concave, define a convex set if they are bound by the inequality

$$
f_{\text {convex }}(\cdot) \leq f_{\text {concave }}(\cdot)
$$

Next, we show that a simple reformulation of problem (5), with removed equality constraints that contain nonlinear terms, does not necessarily yield a convex problem.

\section{A. Compact form reformulation}

Problem (5) can be written in a compact form, with fewer optimization variables, by expressing variables from equality constraints and replacing their occurrences in the problem. Hence, $P_{\mathrm{M}}$ and $P_{\mathrm{G}}$ can be expressed from (5b) and (5c), leading to a problem with just two optimization variables

$$
\begin{aligned}
& \min _{E_{\mathrm{B}}, P_{\mathrm{brk}}} L(\cdot)=\int_{0}^{t_{f}}\left(P_{\mathrm{d}}(\cdot)+P_{\mathrm{brk}}\right) d t \\
& \text { s.t. } \\
& E_{\mathrm{B}}(0)=E_{\mathrm{B}}\left(t_{f}\right)=E_{\mathrm{B} 0} \\
& E_{\mathrm{B}} \in\left[E_{\mathrm{Bmin}}, E_{\mathrm{Bmax}}\right] \\
& -\frac{d E_{\mathrm{B}}}{d t} \in\left[P_{\mathrm{Bmin}}(\cdot), P_{\mathrm{B} \max }(\cdot)\right] \\
& \frac{d E_{\mathrm{B}}}{d t}+P_{\mathrm{Bd}}(\cdot)+P_{\mathrm{Md}}(\cdot)+P_{\mathrm{brk}}+P_{\mathrm{V}}+P_{\mathrm{A}} \\
& P_{\mathrm{brk}} \geq \max \left\{0, P_{\mathrm{Mmin}}-P_{\mathrm{V}}\right\}
\end{aligned}
$$

where the dissipative terms have been gathered in

$$
P_{\mathrm{d}}(\cdot)=P_{\mathrm{Gd}}(\cdot)+P_{\mathrm{Bd}}(\cdot)+P_{\mathrm{Md}}(\cdot)+v F_{\mathrm{Vd}}+P_{\mathrm{A}} .
$$

The function $P_{\mathrm{Gd}}\left(P_{\mathrm{G}}(\cdot), \sigma_{\mathrm{G}}\right)$ is convex, since $P_{\mathrm{Gd}}(\cdot)$ is convex and nondecreasing in $P_{\mathrm{G}}(\cdot)$ and the input argument $P_{\mathrm{G}}(\cdot)$ is a convex function, as expressed from $(5 \mathrm{c})$. It follows that the objective $L(\cdot)$ is a convex function, although reformulated to minimize dissipative energy instead of fuel energy. This function is obtained by removing the difference in potential energy

$$
L(\cdot)=J(\cdot)-m g\left(h\left(t_{f}\right)-h(0)\right)
$$

which does not affect the results. Here, $h$ is the road altitude. It is evident that the optimization outcome will not change even if $P_{\mathrm{A}}$ and $v F_{\mathrm{Vd}}$ are removed from $P_{\mathrm{d}}(\cdot)$. However, these are kept in the problem for resemblance with the strategy discussed later, in Section IV.

Yet, the problem (7) is still not convex, due to the nonconvex set $(7 \mathrm{e})$ that does not satisfy the convexity requirement (6).

\section{B. Convex relaxation}

The idea of convex relaxation is to create a convex superset of the non-convex set, such that the minimum of the relaxed convex problem is the global minimum of the original nonconvex problem. A straightforward way is to introduce two additional optimization variables, $x_{\mathrm{B}}, x_{\mathrm{M}}$, that replace the nonlinear terms in $(7 \mathrm{e})$, yielding

$$
\begin{aligned}
& \min _{E_{\mathrm{B}}, P_{\mathrm{brk}}, x_{\mathrm{B}}, x_{\mathrm{M}}} L(\cdot)=\int_{0}^{t_{f}}\left(P_{\mathrm{d}}(\cdot)+P_{\mathrm{brk}}\right) d t \\
& \text { s.t. }(7 \mathrm{~b}),(7 \mathrm{c}),(7 \mathrm{~d}),(7 \mathrm{f}), \\
& x_{\mathrm{B}} \geq P_{\mathrm{Bd}}(\cdot) \\
& x_{\mathrm{M}} \geq P_{\mathrm{Md}}(\cdot) \\
& \frac{d E_{\mathrm{B}}}{d t}+x_{\mathrm{B}}+x_{\mathrm{M}}+P_{\mathrm{brk}}+P_{\mathrm{V}}+P_{\mathrm{A}} \in\left[0, \sigma_{G} P_{\mathrm{Gmax}}\right]
\end{aligned}
$$

with

$$
P_{\mathrm{d}}(\cdot)=P_{\mathrm{Gd}}(\cdot)+x_{\mathrm{B}}+x_{\mathrm{M}}+v F_{\mathrm{Vd}}+P_{\mathrm{A}} .
$$

It has been logically reasoned in [12], [13] that the optimal solution of the relaxed convex problem (10) yields the optimal solution of the original non-convex problem, as any other solution wastes energy, and therefore, cannot be optimal. A more rigorous proof has been provided by [16] for slightly different problem formulation and stricter monotonicity conditions.

Proposition 1. Given the conditions 1) - 4) listed in Section II, the global minimum of problem (7) can be obtained by solving the relaxed problem (10). Thereby, a sufficient condition for obtaining the minimum of problem (7) is that the inequality constraints (10b) and (10c) of the relaxed problem (10) hold with equality at the optimum.

Proof. Suppose that an optimal solution of problem (10) is found that can be expressed as

$$
\begin{aligned}
& x_{\mathrm{B}}^{*}=P_{\mathrm{Bd}}^{*}(\cdot)+\delta_{\mathrm{B}} \\
& x_{\mathrm{M}}^{*}=P_{\mathrm{Md}}^{*}(\cdot)+\delta_{\mathrm{M}}
\end{aligned}
$$

where $\delta_{\mathrm{B}} \geq 0, \delta_{\mathrm{M}} \geq 0, \forall t \in\left[0, t_{f}\right]$ are slack variables. Then, it is possible to construct an alternative suboptimal solution with the same battery energy and braking trajectory, $\tilde{E}_{\mathrm{B}}=$ $E_{\mathrm{B}}^{*}, \tilde{P}_{\text {brk }}=P_{\text {brk }}^{*}$, but with tight constraints $(10 \mathrm{~b})$ and $(10 \mathrm{c})$,

$$
\begin{aligned}
& \tilde{x}_{\mathrm{B}}=x_{\mathrm{B}}^{*}-\delta_{\mathrm{B}} \\
& \tilde{x}_{\mathrm{M}}=x_{\mathrm{M}}^{*}-\delta_{\mathrm{M}} .
\end{aligned}
$$

A new slack variable is introduced

$$
\delta_{\mathrm{G}}=\max \left\{0, \delta_{\mathrm{B}}+\delta_{\mathrm{M}}-P_{\mathrm{G}}^{*}(\cdot)\right\}
$$


where

$$
P_{\mathrm{G}}^{*}(\cdot)=\frac{d E_{\mathrm{B}}^{*}}{d t}+x_{\mathrm{B}}^{*}+x_{\mathrm{M}}^{*}+P_{\mathrm{brk}}^{*}+P_{\mathrm{V}}+P_{\mathrm{A}},
$$

which ensures a feasible solution

$$
\tilde{P}_{\mathrm{G}}(\cdot)=\frac{d E_{\mathrm{B}}^{*}}{d t}+\tilde{x}_{\mathrm{B}}+\tilde{x}_{\mathrm{M}}+P_{\text {brk }}^{*}+P_{\mathrm{V}}+P_{\mathrm{A}}+\delta_{\mathrm{G}} \geq 0 .
$$

It follows that

$$
\tilde{P}_{\mathrm{G}}(\cdot)-P_{\mathrm{G}}^{*}(\cdot)=\delta_{\mathrm{B}}+\delta_{\mathrm{M}}-\max \left\{0, \delta_{\mathrm{B}}+\delta_{\mathrm{M}}-P_{\mathrm{G}}^{*}(\cdot)\right\} \leq 0
$$

for any $P_{\mathrm{G}}^{*}(\cdot) \geq 0$.

1) Time instances with strictly positive EGU power: Consider the sets

$$
\begin{aligned}
& \mathcal{T}_{0}=\left\{t \in\left[0, t_{f}\right] \mid P_{\mathrm{G}}^{*}(\cdot)=0\right\} \\
& \mathcal{T}_{1}=\left\{t \in\left[0, t_{f}\right] \backslash \mathcal{T}_{0}\right\}
\end{aligned}
$$

where $\mathcal{T}_{0}$ is the set of time instances where the engine is off or idling, and $\mathcal{T}_{1}$ gathers the remaining time instances. It follows that $\forall t \in \mathcal{T}_{1}, \tilde{P}_{\mathrm{G}}(\cdot)<P_{\mathrm{G}}^{*}(\cdot)$, which due to the monotonicity of $P_{\mathrm{Gd}}\left(P_{\mathrm{G}}, \cdot\right)$ implies that $\tilde{L}(\cdot)<L^{*}(\cdot)$. Hence, $L^{*}(\cdot)$, rather than $\tilde{L}(\cdot)$, is suboptimal, while minimum of $\tilde{L}(\cdot)$ is obtained for $\delta_{\mathrm{B}}=\delta_{\mathrm{M}}=0, \forall t \in \mathcal{T}_{1}$, which consequently gives $\delta_{\mathrm{G}}=0, \forall t \in \mathcal{T}_{1}$.

2) Time instances under electrical operation: Minimizing the cost for $t \in \mathcal{T}_{0}$ does not bring direct incentive that necessitates $\delta_{\mathrm{B}}=\delta_{\mathrm{M}}=0$, since in this interval $\tilde{L}(\cdot)=L^{*}(\cdot)$ regardless of the slack variables' values. To investigate the effect of the slack variables, the cost function is reformulated by splitting the integral in parts

$$
\begin{aligned}
L^{*}(\cdot) & =\int_{t \in \mathcal{T}_{0}}\left(\frac{d E_{\mathrm{B}}^{*}}{d t}+P_{\mathrm{d}}^{*}(\cdot)+P_{\mathrm{brk}}^{*}\right) d t \\
& +\int_{t \in \mathcal{T}_{1}}\left(\frac{d E_{\mathrm{B}}^{*}}{d t}+P_{\mathrm{d}}^{*}(\cdot)+P_{\mathrm{brk}}^{*}\right) d t
\end{aligned}
$$

where battery energy is conserved according to (7b), i.e.

$$
\int_{0}^{t_{f}} d E_{\mathrm{B}}^{*}=\int_{t \in \mathcal{T}_{0}} d E_{\mathrm{B}}^{*}+\int_{t \in \mathcal{T}_{1}} d E_{\mathrm{B}}^{*}=0 .
$$

Since the first integral in (13) corresponds to purely electrical propulsion that does not affect optimization cost directly, it is beneficial to maximize the term $\int_{t \in \mathcal{T}_{0}} d E_{\mathrm{B}}^{*}$, which according to (14) yields decreased value $\int_{t \in \mathcal{T}_{1}} d E_{\mathrm{B}}^{*}$, and thus decreased optimization cost $L^{*}(\cdot)$. In practise, this means that battery energy is maximized by recuperating braking energy as much as possible. Solving

$$
\begin{gathered}
\max _{\delta_{\mathrm{B}} \geq 0, \delta_{\mathrm{M}} \geq 0} \int_{\mathcal{T}_{0}} d E_{\mathrm{B}}^{*}=-\int_{\mathcal{T}_{0}}\left(\tilde{x}_{\mathrm{B}}+\tilde{x}_{\mathrm{M}}+P_{\mathrm{V}}+P_{\mathrm{A}}\right) d t \\
-\int_{\mathcal{T}_{0}}\left(\delta_{\mathrm{B}}+\delta_{\mathrm{M}}+P_{\text {brk }}^{*}\right) d t \\
\text { s.t. } E_{\mathrm{B}}^{*} \leq E_{\mathrm{B} \max }, \quad \frac{d E_{\mathrm{B}}^{*}}{d t} \leq-P_{\mathrm{Bmin}}(\cdot)
\end{gathered}
$$

implies that optimum is obtained when $\delta_{\mathrm{B}}=\delta_{\mathrm{M}}=0, \forall t \in$ $\mathcal{T}_{0}$. Hence, a sufficient condition for obtaining the solution of problem (7) is $\delta_{\mathrm{B}}=\delta_{\mathrm{M}}=0, \forall t \in\left[0, t_{f}\right]$, although it is not necessary that the slack variables are zero when a constraint in (15b) is activated and wasting away braking energy is unavoidable. Then, dissipating the braking energy on the braking pads or on the slack variables is interchangeable in the view of problem (15). At these time instances, the solution of the relaxed problem (10) may give the optimal battery energy trajectory of problem (7), but with $\delta_{\mathrm{B}}+\delta_{\mathrm{M}}>$ 0 . Thereafter, the optimal braking power can be computed as

$$
\tilde{P}_{\mathrm{brk}}=\delta_{\mathrm{B}}+\delta_{\mathrm{M}}+P_{\mathrm{brk}}^{*}
$$

after the optimization is finished, which consequently is the optimal solution of problem (7), obtained by solving the relaxed problem (10).

Similar convex relaxations have been undertaken in [12], [13], [24], [6], [15], [16], [17], [18] for problems of combined sizing and control of electrified powertrains. Common in these studies is that braking power is removed from the problem. Instead, the mechanical EM power or torque is kept as optimization variable. Should braking power be needed after the optimization is finished, it would be computed according to (16).

\section{CONVEX RELAXATION FOR FORWARD SIMULATION}

In the forward simulation approach, the vehicle speed remains a decision variable. The resulting optimization problem is inherently more complex and, therefore, researchers have taken several steps to reduce the computational burden. A typical approach is to sample in space, rather than time, wherein the number of samples is kept low by, e.g., using a shorter receding horizon, which is a common strategy for control of conventional vehicles [31], [32], [33], [34], [3]. For electrified vehicles it is of interest to employ longer prediction horizon, while the computational burden is instead relaxed by simplifying the powertrain model. Batteries and electric machines are commonly approximated by constant efficiencies and engine losses are described by affine relations [10], [35].

\section{A. Component models}

Data for the EGU and EM model is obtained by performing measurements under stationary conditions. The original data, together with fitted models, affine for the EGU losses and piecewise affine for the EM losses,

$$
\begin{aligned}
& P_{\mathrm{Gd}}(\cdot)=a_{0} \sigma_{\mathrm{G}}+\left(a_{1}-1\right) P_{\mathrm{G}} \\
& P_{\mathrm{Md}}(\cdot)= \begin{cases}b_{11} v+b_{12} P_{\mathrm{M}}, & P_{\mathrm{M}}<0 \\
b_{21} v+b_{22} P_{\mathrm{M}}, & P_{\mathrm{M}} \geq 0\end{cases}
\end{aligned}
$$

are depicted in Fig. 2.

The battery losses are modeled by a quadratic function of the internal battery power

$$
P_{\mathrm{Bd}}(\cdot)=\frac{R}{U^{2}} P_{\mathrm{B}}^{2}=\frac{R}{U^{2}}\left(\frac{d E_{\mathrm{B}}}{d s} v\right)^{2}
$$

where the open circuit voltage $U$ and inner resistance $R$ are known scalar values. Thus, the battery power limits $P_{\mathrm{Bmin}}, P_{\mathrm{Bmax}}$ also become known scalar values. 

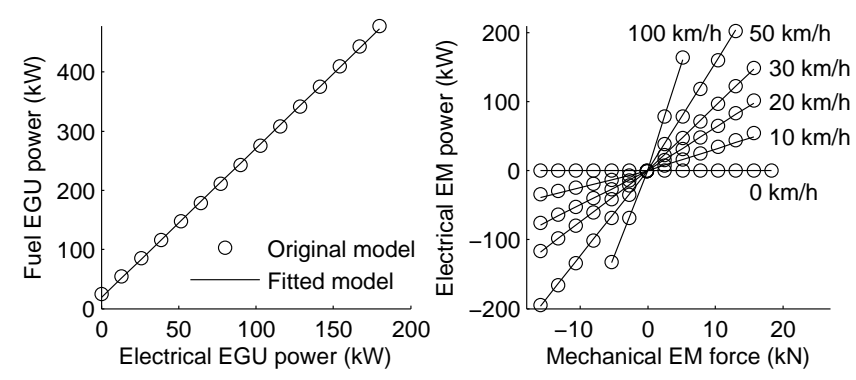

Fig. 2. Original and fitted models of the EGU (left) and EM (right). The EM electrical power is plotted vs. produced longitudinal vehicle force, for several values of vehicle speed.

The battery dynamics are expressed as

$$
\frac{d E_{\mathrm{B}}}{d s} v=-P_{\mathrm{B}}
$$

where sampling in space is used instead of sampling in time.

\section{B. Convex modeling}

The first convex modeling step is to perform variable changes. As suggested by [7], vehicle kinetic energy is used instead of longitudinal speed and forces are used instead of powers

$$
\begin{aligned}
& E_{\mathrm{V}}=\frac{m v^{2}}{2}, F_{\mathrm{brk}}=\frac{P_{\text {brk }}}{v}, F_{\mathrm{V}}(\cdot)=\frac{P_{\mathrm{V}}(\cdot)}{v}, \\
& F_{\mathrm{Md}}(\cdot)=\frac{P_{\mathrm{Md}}(\cdot)}{v}, y_{\mathrm{B}}=\frac{x_{\mathrm{B}}}{v}, y_{\mathrm{M}}=\frac{x_{\mathrm{M}}}{v} .
\end{aligned}
$$

Accordingly, the power limits of the components will translate to force limits. The force limits are linearized about the reference kinetic energy $E_{\mathrm{V} r}$,

$$
F_{\mathrm{Xlim}}(\cdot)=\frac{P_{\mathrm{Xlim}}\left(v_{r}\right)}{2 v_{r}}\left(3-\frac{E_{\mathrm{V}}}{E_{\mathrm{V} r}}\right), \quad \mathrm{X} \in\{\mathrm{B}, \mathrm{M}, \mathrm{G}\}
$$

yielding affine functions $F_{\text {Xlim }}(\cdot)$ with $\lim =\{\min , \max \}$. The error due to these approximations is generally small, since powertrain components are often not operated at peak power. Nevertheless, the error can be further decreased by iterating the optimization and using the optimal velocity as a reference in the succeeding iteration.

The optimization problem, applicable strictly to vehicles in motion, i.e. $v_{\min }>0$, can now be summarized as

$$
\min _{E_{\mathrm{V}}, E_{\mathrm{B}}, F_{\text {brk }}, y_{\mathrm{B}}, y_{\mathrm{M}}} L(\cdot)=\int_{0}^{s_{f}}\left(F_{\mathrm{d}}(\cdot)+a_{1} F_{\text {brk }}\right) d s
$$

s.t. (7b), (7c),

$$
\begin{aligned}
& y_{\mathrm{B}} \geq \frac{R}{U^{2}}\left(\frac{d E_{\mathrm{B}}}{d s}\right)^{2} \sqrt{\frac{2 E_{\mathrm{V}}}{m}} \\
& y_{\mathrm{M}} \geq F_{\mathrm{Md}}(\cdot) \\
& \sqrt{\frac{m}{2}} \int_{0}^{s_{f}} \frac{d s}{\sqrt{E_{\mathrm{V}}}} \leq t_{\max } \\
& E_{\mathrm{V}}(0)=E_{\mathrm{V}}\left(s_{f}\right)=m v_{0}^{2} / 2 \\
& E_{\mathrm{V}} \in\left[m v_{\min }^{2} / 2, m v_{\max }^{2} / 2\right] \\
& -\frac{d E_{\mathrm{B}}}{d s} \in\left[F_{\mathrm{B} \min }(\cdot), F_{\mathrm{B} \max }(\cdot)\right]
\end{aligned}
$$

$$
\begin{aligned}
& \frac{d E_{\mathrm{B}}}{d s}+y_{\mathrm{B}}+y_{\mathrm{M}}+F_{\mathrm{brk}}+F_{\mathrm{V}}(\cdot)+P_{\mathrm{A}} \sqrt{\frac{m}{2 E_{\mathrm{V}}}} \\
& \in\left[0, \sigma_{\mathrm{G}} F_{\mathrm{Gmax}}(\cdot)\right] \\
& F_{\text {brk }} \geq \max \left\{0, F_{\mathrm{Mmin}}(\cdot)-F_{\mathrm{V}}(\cdot)\right\} \\
& F_{\text {brk }} \leq F_{\text {Mmax }}(\cdot)-F_{\mathrm{V}}(\cdot)
\end{aligned}
$$

where the longitudinal vehicle force

$$
\begin{aligned}
& F_{\mathrm{V}}(\cdot)=\frac{d E_{\mathrm{V}}}{d s}+m g \sin \alpha+F_{\mathrm{Vd}}(\cdot) \\
& F_{\mathrm{Vd}}(\cdot)=\frac{\rho_{a} c_{d} A_{f}}{m} E_{\mathrm{V}}+m g c_{r} \cos \alpha
\end{aligned}
$$

is affine in $E_{\mathrm{V}}$. The dissipative forces

$$
\begin{aligned}
& F_{\mathrm{d}}(\cdot)=\left(a_{0} \sigma_{\mathrm{G}}+a_{1} P_{\mathrm{A}}\right) \sqrt{\frac{m}{2 E_{\mathrm{V}}}}+a_{1}\left(y_{\mathrm{B}}+y_{\mathrm{M}}+F_{\mathrm{Vd}}(\cdot)\right) \\
& F_{\mathrm{Md}}(\cdot)=\frac{P_{\mathrm{Md}}(\cdot)}{v}= \\
& \quad \max \left\{b_{11}+b_{12}\left(F_{\mathrm{V}}(\cdot)+F_{\text {brk }}\right), b_{21}+b_{22}\left(F_{\mathrm{V}}(\cdot)+F_{\text {brk }}\right)\right\}
\end{aligned}
$$

are convex in $y_{\mathrm{B}}, y_{\mathrm{M}}, E_{\mathrm{V}}$, respectively. The optimization objective (23a) is also convex, but the problem is not convex due to the non-convex right hand side of (23b) and the constraint (23h) that does not satisfy the requirement (6).

\section{Convex relaxation}

The problem (23) can be made convex by introducing a new variable $y_{t}$, function of distance,

$$
y_{t}=\frac{1}{v}=\sqrt{\frac{m}{2 E_{\mathrm{V}}}}
$$

which depicts the time needed to drive one meter of distance. Since the right hand side of (26) is nonlinear, the constraint is relaxed, yielding the convex optimization problem

$$
\min _{E_{\mathrm{V}}, E_{\mathrm{B}}, F_{\mathrm{brk}}, y_{\mathrm{B}}, y_{\mathrm{M}}, y_{t}} L(\cdot)=\int_{0}^{s_{f}}\left(F_{\mathrm{d}}(\cdot)+a_{1} F_{\mathrm{brk}}\right) d s
$$

$$
\text { s.t. (7b), (7c), (23c)-(23g), (23i), (23j), }
$$

$y_{t} \geq \sqrt{\frac{m}{2 E_{\mathrm{V}}}}$

$y_{\mathrm{B}} \geq \frac{R}{U^{2}}\left(\frac{d E_{\mathrm{B}}}{d s}\right)^{2} \frac{1}{y_{t}}$

$$
\frac{d E_{\mathrm{B}}}{d s}+y_{\mathrm{B}}+y_{\mathrm{M}}+F_{\mathrm{brk}}+F_{\mathrm{V}}(\cdot)+P_{\mathrm{A}} y_{t} \in\left[0, \sigma_{\mathrm{G}} F_{\mathrm{G} m a x}(\cdot)\right]
$$

where

$$
F_{\mathrm{d}}(\cdot)=y_{t}\left(a_{0} \sigma_{\mathrm{G}}+a_{1} P_{\mathrm{A}}\right)+a_{1}\left(y_{\mathrm{B}}+y_{\mathrm{M}}+F_{\mathrm{Vd}}(\cdot)\right) .
$$

However, it is not obvious that the solution of the relaxed problem (27) returns the global optimum of problem (23). Indeed, there is an incentive for (27b) to hold with inequality, since larger $y_{t}$ may decrease battery losses in $(27 \mathrm{c})$.

Proposition 2. The global minimum of problem (23) can be obtained by solving the relaxed problem (27), if the optimal battery power trajectory $P_{B}^{*}$ obtained by solving problem (27) satisfies

$$
P_{\mathrm{Bd}}\left(P_{\mathrm{B}}^{*}\right) \leq \sigma_{\mathrm{G}} \frac{a_{0}}{a_{1}}+P_{\mathrm{A}}, \quad \forall s \in\left[0, s_{f}\right] .
$$


Thereby, a sufficient condition for obtaining the solution of (23) by solving (27) is

$$
P_{\mathrm{Bd}}\left(P_{\mathrm{Blim}}\right) \leq \sigma_{\mathrm{G}} \frac{a_{0}}{a_{1}}+P_{\mathrm{A}}, \quad \lim =\{\min , \max \}
$$

for a given engine on/off sequence $\sigma_{E}, \forall s \in\left[0, s_{f}\right]$.

Proof. Disagreement between the optimums of the relaxed and non-relaxed problems may occur, if there exists a positive slack variable $\delta_{t}>0$ that satisfies (27b) with equality, for which the cost of the relaxed problem is lower than the cost of the non-relaxed problem. Denoting with $y_{t}^{*}=\tilde{y}_{t}+\delta_{t}$ the solution of the relaxed problem (27), the undesirable scenario takes place when

$$
\begin{aligned}
\left(\tilde{y}_{t}+\delta_{t}\right)\left(a_{0} \sigma_{\mathrm{G}}+a_{1} P_{\mathrm{A}}\right)+\frac{R}{U^{2}}\left(\frac{d E_{\mathrm{B}}^{*}}{d s}\right)^{2} \frac{a_{1}}{\tilde{y}_{t}+\delta_{t}} \\
<\tilde{y}_{t}\left(a_{0} \sigma_{\mathrm{G}}+a_{1} P_{\mathrm{A}}\right)+\frac{R}{U^{2}}\left(\frac{d E_{\mathrm{B}}^{*}}{d s}\right)^{2} \frac{a_{1}}{\tilde{y}_{t}}
\end{aligned}
$$

or equivalently, when there exists $\delta_{t}$ such that

$$
\begin{aligned}
0<\delta_{t} & \leq y_{t}\left(\frac{a_{1} R\left(\frac{d E_{\mathrm{B}}^{*}}{d s}\right)^{2}}{U^{2} y_{t}^{2}\left(a_{0} \sigma_{\mathrm{G}}+a_{1} P_{\mathrm{A}}\right)}-1\right) \\
& =y_{t}\left(\frac{a_{1} P_{\mathrm{Bd}}(\cdot)}{a_{0} \sigma_{\mathrm{G}}+a_{1} P_{\mathrm{A}}}-1\right) .
\end{aligned}
$$

A positive slack variable $\delta_{t}$ will not exist if the last term in parentheses in (30) is less than or equal to zero. Thus, if (28) is satisfied, the solution of the non-relaxed problem can be obtained by solving the relaxed problem.

Using $y_{t}$ instead of $E_{\mathrm{V}}$ in (23d) gives a milder condition than (28). However, investigation of this scenario is not pursued in this paper.

\section{NumericAl EXAMPLE}

The backward and forward simulation approaches are applied here to a predictive cruise control of a hybrid electric bus with a prediction horizon of $21 \mathrm{~km}$. The EGU and EM model have been given in Fig. 2, while the remaining vehicle parameters are listed in Table I. In the backward simulation approach it is assumed that the vehicle velocity is equal to the reference velocity set by the driver, $v_{r}=$ $70 \mathrm{~km} / \mathrm{h}, \forall t \in\left[0, t_{f}\right]$. In the forward simulation approach the vehicle velocity is allowed to vary within $v_{r} \pm 10 \mathrm{~km} / \mathrm{h}$, but the initial and final velocity are enforced to be equal to the reference, $v(0)=v\left(s_{f}\right)=v_{r}$, and the total travel time is not allowed to exceed the driving time in backward simulation, $t_{\max }=21 / 70=0.3 \mathrm{~h}$.

The problem is written in CVX modeling language [36], [37] and solved with ECOS [20]. First order Euler discretization is used with sampling interval of $100 \mathrm{~m}$ for the forward simulation approach and $5.14 \mathrm{~s}$ for the backward simulation approach (giving equal number of samples in both approaches).

The optimization is carried on with $\sigma_{\mathrm{G}}=1, \forall s \in\left[0, s_{f}\right]$, with which condition (29) is satisfied $\forall s \in\left[0, s_{f}\right]$. The
TABLE I

VEHICLE SPECIFICATIONS.

\begin{tabular}{lll}
\hline$A_{f}=7.54 \mathrm{~m}^{2}$ & $R=0.091 \Omega$ & $a_{0}=19 \mathrm{~kW}$ \\
$c_{d}=0.7$ & $U=330 \mathrm{~V}$ & $a_{1}=2.52$ \\
$c_{r}=0.007$ & $P_{\mathrm{Bmax}}=92.4 \mathrm{~kW}$ & $b_{11}=2.13 \mathrm{~N}$ \\
$\rho_{a}=1.184 \mathrm{~kg} / \mathrm{m}^{3}$ & $P_{\mathrm{Bmin}}=-92.4 \mathrm{~kW}$ & $b_{12}=-0.113$ \\
$P_{\mathrm{A}}=7 \mathrm{~kW}$ & $E_{\mathrm{B} \min }=2.1 \mathrm{kWh}$ & $b_{21}=5.63 \mathrm{~N}$ \\
$m=15.95 \mathrm{t}$ & $E_{\mathrm{B} \max }=6.3 \mathrm{kWh}$ & $b_{22}=0.123$ \\
\hline
\end{tabular}

TABLE II

OPTIMIZATION RESULTS.

\begin{tabular}{lll}
\hline Optimization approach: & Forward sim. & Backward sim. \\
Fuel consumption & $23.98 \mathrm{l} / 100 \mathrm{~km}$ & $24.35 \mathrm{l} / 100 \mathrm{~km}$ \\
Drag and rolling dissipation & $13.49 \mathrm{kWh}$ & $13.34 \mathrm{kWh}$ \\
Available braking energy & $5.51 \mathrm{kWh}$ & $6.27 \mathrm{kWh}$ \\
Recuperated braking energy & $100 \%$ & $98.24 \%$ \\
Max. battery dissipative power & $3.35 \mathrm{~kW}$ & $2.96 \mathrm{~kW}$ \\
Average optimization time $^{a}$ & $0.4 \mathrm{~s}$ & $0.2 \mathrm{~s}$ \\
\hline
\end{tabular}

${ }^{a} \mathrm{PC}$ with $2.67 \mathrm{GHz}$ dual-core processor and $4 \mathrm{~GB}$ RAM.

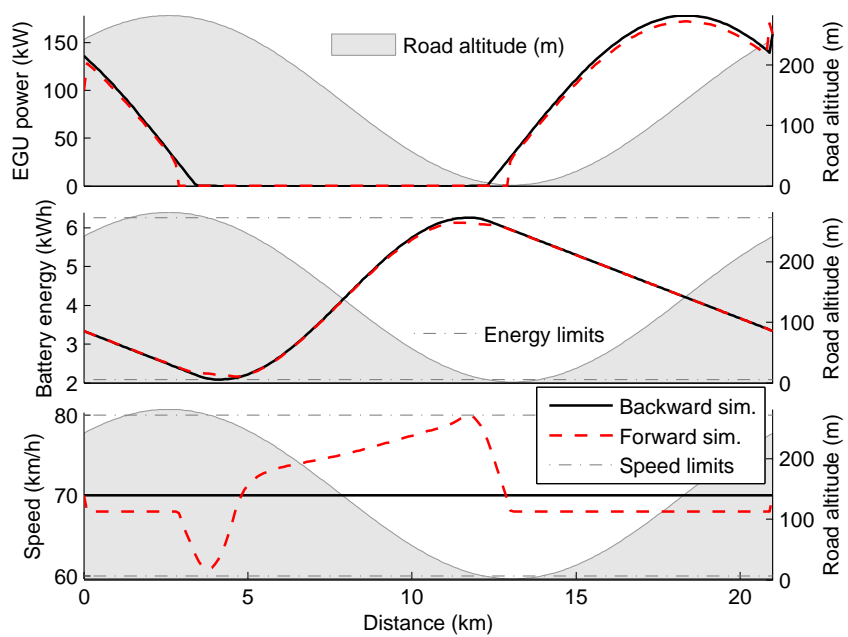

Fig. 3. Optimal control and state trajectories for the backward and forward simulation approach. Road altitude is depicted on the right axes.

optimization results are depicted in Fig. 3, and summarized in Table II. The results indicate that when speed is optimally controlled the vehicle accelerates in the downhill segment, thus accumulating kinetic energy, which is later used to drive the vehicle in the uphill segment. Consequently, the available braking energy is decreased by $12 \%$ (compared to the backward simulation), and the vehicle is capable of recuperating all the energy, which is stored in the battery for a later use. The optimization time is less than $1 \mathrm{~s}$, but could be further decreased by using a dedicated solver and feeding the problem directly to the solver, in a sparse matrix form.

The top plot of Fig. 3 indicates that there is no need to idle the engine when $P_{\mathrm{G}} \approx 0$. Re-optimizing with the engine turned off should be performed with care. The sufficient condition (29) will not be satisfied when engine is turned off, although it could be expected, as depicted in Table II, that the necessary condition (28) would still be satisfied.

\section{CONCLUSION}

This paper provides convex relaxation techniques for the optimal power-split control of an HEV. Two optimization 
approaches are considered, first, a backward simulation approach, where vehicle speed is equal to the reference, and second, a forward simulation approach, where vehicle speed is a decision variable. It is shown that in the first case the global optimum can be obtained by solving a relaxed convex problem, while in the second case a condition is provided, which when satisfied, guarantees that the solution of the nonrelaxed problem can be obtained by solving a relaxed convex problem.

Although this paper considered an $\mathrm{HEV}$ in a series powertrain configuration, it is straightforward to extend the strategy to energy management of hybrid vehicles in other powertrain configurations, as well as to conventional and electric vehicles. Furthermore, it is straightforward to extend the strategy to problems with simultaneous powertrain design and control of vehicles [24]. Detailed investigation of these alternatives will be pursued in future studies.

\section{REFERENCES}

[1] T. Lipp and S. Boyd, "Minimum-time speed optimization along a fixed path," International Journal of Control, vol. 87, no. 6, pp. 1297-1311, 2014.

[2] R. de Castro, M. Tanelli, R. E. Araújo, and S. M. Savaresi, "Minimumtime path following in highly redundant electric vehicles," in IFAC World Congress, Cape Town, South Africa, 2014, pp. 3918-3923.

[3] C. Kirches, H. G. Bock, J. P. Schlöder, and S. Sager, "Mixed-integer NMPC for predictive cruise control of heavy-duty trucks," in European Control Conference, Zürich, Switzerland, 2013, pp. 4118-4123.

[4] L. Guzzella and A. Sciarretta, Vehicle Propulsion Systems, 3rd ed. Berlin, Heidelberg: Springer, Verlag, 2013.

[5] E. D. Tate and S. P. Boyd, "Finding ultimate limits of performance for hybrid electric vehicles," in SAE Technical Paper 2000-01-3099, 2000.

[6] X. Hu, N. Murgovski, L. Johannesson, and B. Egardt, "Energy efficiency analysis of a series plug-in hybrid electric bus with different energy management strategies and battery sizes," Applied Energy, vol. 111, no. 0, pp. 1001-1009, 2013.

[7] N. Murgovski, X. Hu, L. Johannesson, and B. Egardt, "Filtering driving cycles for assessment of electrified vehicles," in Workshop for new energy vehicle dynamic system and control technology, Beijing, China, 2013.

[8] M. Koot, J. T. B. A. Kessels, B. de Jager, W. P. M. H. Heemels, P. P. J. van den Bosch, and M. Steinbuch, "Energy management strategies for vehicular electric power systems," IEEE Transactions on Vehicular Technology, vol. 54, no. 3, pp. 771-782, 2005.

[9] R. Beck, A. Bollig, and D. Abel, "Comparison of two real-time predictive strategies for the optimal energy management of a hybrid electric vehicle," Oil \& Gas Science and Technology, vol. 62, no. 4, pp. 635-643, 2007.

[10] D. Ambühl and L. Guzzella, "Predictive reference signal generator for hybrid electric vehicles," IEEE Transactions on Vehicular Technology, vol. 58, no. 9, pp. 4730-4740, 2009.

[11] N. Murgovski, X. Hu, and B. Egardt, "Computationally efficient energy management of a planetary gear hybrid electric vehicle," in IFAC World Congress, Cape Town, South Africa, 2014.

[12] N. Murgovski, L. Johannesson, J. Hellgren, B. Egardt, and J. Sjöberg, "Convex optimization of charging infrastructure design and component sizing of a plug-in series HEV powertrain," in IFAC World Congress, Milan, Italy, 2011.

[13] N. Murgovski, L. Johannesson, J. Sjöberg, and B. Egardt, "Component sizing of a plug-in hybrid electric powertrain via convex optimization," Mechatronics, vol. 22, no. 1, pp. 106-120, 2012.

[14] M. Pourabdollah, N. Murgovski, A. Grauers, and B. Egardt, "Optimal sizing of a parallel PHEV powertrain," IEEE Transactions on Vehicular Technology, vol. 62, no. 6, pp. 2469-2480, 2013.

[15] N. Murgovski, L. Johannesson, and B. Egardt, "Optimal battery dimensioning and control of a CVT PHEV powertrain," IEEE Transactions on Vehicular Technology, vol. 63, no. 5, pp. 2151-2161, 2014.
[16] B. Egardt, N. Murgovski, M. Pourabdollah, and L. Johannesson, "Electromobility studies based on convex optimization: Design and control issues regarding vehicle electrification," IEEE Control Systems Magazine, vol. 34, no. 2, pp. 32-49, 2014.

[17] X. Hu, N. Murgovski, L. Johannesson, and B. Egardt, "Comparison of three electrochemical energy buffers applied to a hybrid bus powertrain with simultaneous optimal sizing and energy management," IEEE Transactions on Intelligent Transportation Systems, vol. 15, no. 3, pp. 1193-1205, 2014.

[18] _ - "Optimal dimensioning and power management of a fuel cell/battery hybrid bus via convex programming," IEEE/ASME Transactions on Mechatronics, vol. 20, no. 1, pp. 457-468, 2015.

[19] X. Hu, L. Johannesson, N. Murgovski, and B. Egardt, "Longevityconscious dimensioning and power management of the hybrid energy storage system for a fuel cell hybrid electric bus," Applied Energy, vol. 137, no. 0, pp. 913-924, 2015.

[20] A. Domahidi, E. Chu, and S. Boyd, "ECOS: An SOCP solver for embedded systems," in European Control Conference (ECC), 2013, pp. 3071-3076.

[21] Y. Labit, D. Peaucelle, and D. Henrion, "SeDuMi interface 1.02: a tool for solving LMI problems with SeDuMi," IEEE International Symposium on Computer Aided Control System Design Proceedings, pp. 272-277, 2002.

[22] K. C. Toh, R. H. Tütüncü, and M. J. Todd, "On the implementation and usage of SDPT3 - a Matlab software package for semidefinitequadratic-linear programming, version 4.0," 2006.

[23] N. Murgovski, L. Johannesson, and J. Sjöberg, "Convex modeling of energy buffers in power control applications," in IFAC Workshop on Engine and Powertrain Control, Simulation and Modeling (E-CoSM), Rueil-Malmaison, Paris, France, 2012.

[24] N. Murgovski, "Optimal powertrain dimensioning and potential assessment of hybrid electric vehicles," Ph.D. dissertation, Chalmers University of Technology, Gothenburg, Sweden, 2012.

[25] M. Neuman, H. Sandberg, B. Wahlberg, and A. Folkesson, "Modelling and control of series HEVs including resistive losses and varying engine efficiency," in SAE International, 2008.

[26] N. Murgovski, L. Johannesson, and J. Sjöberg, "Engine on/off control for dimensioning hybrid electric powertrains via convex optimization," IEEE Transactions on Vehicular Technology, vol. 62, no. 7, pp. 29492962, 2013.

[27] P. Elbert, T. Nüesch, A. Ritter, N. Murgovski, and L. Guzzella, "Engine on/off control for the energy management of a serial hybrid electric bus via convex optimization,' IEEE Transactions on Vehicular Technology, vol. 63 , no. 8, pp. 3549-3559, 2014

[28] A. Sciarretta and L. Guzzella, "Control of hybrid electric vehicles," IEEE Control Systems Magazine, vol. 27, no. 2, pp. 60-70, 2007.

[29] T. van Keulen, B. de Jager, D. Foster, and M. Steinbuch, "Velocity trajectory optimization in hybrid electric trucks," in American Control Conference, Marriott Waterfront, Baltimore, MD, USA, 2010, pp. 5074-5079.

[30] S. Boyd and L. Vandenberghe, Convex Optimization. Cambridge University Press, 2004.

[31] S. Terwen, M. Back, and V. Krebs, "Predictive powertrain control for heavy duty trucks," in Proc. IFAC Symposium on Advances in Automotive Control, Salerno, Italy, 2004, pp. 105-110.

[32] A. Fröberg and L. Nielsen, "Optimal control utilizing analytical solutions for heavy truck cruise control," Dept. of Electrical Engineering, Linköping University, Sweden, Tech. Rep. LiTH-ISY-R-2842, 2008.

[33] E. Hellström, M. Ivarsson, J. Åslund, and L. Nielsen, "Look-ahead control for heavy trucks to minimize trip time and fuel consumption," Control Engineering Practice, vol. 17, no. 2, pp. 245-254, 2009.

[34] B. Passenberg, P. Kock, and O. Stursberg, "Combined time and fuel optimal driving of trucks based on a hybrid model," in European Control Conference, Budapest, Hungary, 2009, pp. 4955-4960.

[35] T. van Keulen, B. de Jager, and M. Steinbuch, "Optimal trajectories for vehicles with energy recovery options," in IFAC World Congress, Milan, Italy, 2011, pp. 3831-3836.

[36] I. CVX Research, "CVX: Matlab software for disciplined convex programming, version 2.0 beta," http://cvxr.com/cvx, 2012.

[37] M. Grant and S. Boyd, "Graph implementations for nonsmooth convex programs," in Recent Advances in Learning and Control, ser. Lecture Notes in Control and Information Sciences, V. Blondel, S. Boyd, and H. Kimura, Eds. Springer-Verlag Limited, 2008, pp. 95-110, http://stanford.edu/ boyd/graph_dep.html. 